\title{
MEASURING THE MATTER DENSITY USING BARYON OSCILLATIONS IN THE SDSS
}

\author{
Will J. Percival, ${ }^{1}$ Robert C. Nichol, ${ }^{1}$ Daniel J. Eisenstein, ${ }^{2}$ David H. Weinberg, ${ }^{3}$ Masataka Fukugita, \\ Adrian C. Pope ${ }^{5,6}$ Donald P. Schneider, ${ }^{7}$ Alex S. Szalay, ${ }^{5}$ Michael S. Vogeley, ${ }^{8}$ Idit Zehavi, ${ }^{9}$ \\ Neta A. Bahcall, ${ }^{10}$ Jon Brinkmann, ${ }^{11}$ Andrew J. Connolly, ${ }^{12}$ Jon Loveday, $^{13}$ and Avery Meiksin ${ }^{14}$ \\ Received 2006 August 30; accepted 2006 November 9
}

\begin{abstract}
We measure the cosmological matter density by observing the positions of baryon acoustic oscillations in the clustering of galaxies in the Sloan Digital Sky Survey (SDSS). We jointly analyze the main galaxies and LRGs in the SDSS DR5 sample, using over half a million galaxies in total. The oscillations are detected with $99.74 \%$ confidence (3.0 $\sigma$ assuming Gaussianity) compared to a smooth power spectrum. When combined with the observed scale of the peaks within the CMB, we find a best-fit value of $\Omega_{M}=0.256_{-0.024}^{+0.029}$ ( $68 \%$ confidence interval) for a flat $\Lambda$ cosmology when marginalizing over the Hubble parameter and the baryon density. This value of the matter density is derived from the locations of the baryon oscillations in the galaxy power spectrum and in the CMB, and does not include any information from the overall shape of the power spectra. This is an extremely clean cosmological measurement, as the physics of the baryon acoustic oscillation production is well understood, and the positions of the oscillations are expected to be independent of systematics such as galaxy bias.
\end{abstract}

Subject headings: cosmological parameters - large-scale structure of universe

\section{INTRODUCTION}

Baryonic acoustic oscillations (BAO) are predicted in the matter distribution with a calibration that depends on $\Omega_{M} h^{2}$ (Silk 1968; Peebles \& Yu 1970; Sunyaev \& Zel'dovich 1970; Bond \& Efstathiou 1984, 1987; Holtzman 1989). The oscillations arise because sound waves in the coupled baryon-photon plasma, after an inflationary epoch, lead to the expansion of the baryonic material in a spherical shell around a small perturbation, reaching a radius $r_{S}\left(z_{*}\right)$, the comoving sound horizon size at recombination, before sound waves are no longer supported within the plasma (Bashinsky \& Bertschinger 2001, 2002). At the high redshifts of interest the vacuum energy can be neglected, and $r_{S}\left(z_{*}\right)$ can be simply written as (Hu \& Sugiyama 1995)

$$
\frac{r_{S}\left(z_{*}\right)}{h^{-1} \mathrm{Mpc}} \equiv \frac{1}{100 \Omega_{m}^{1 / 2}} \int_{0}^{a_{*}} \frac{c_{S}}{\left(a+a_{\mathrm{eq}}\right)^{1 / 2}} d a .
$$

The expansion factor $a \equiv(1+z)^{-1}$, and $a_{*}, a_{\mathrm{eq}}$ are the values at recombination and matter-radiation equality, respectively. Thus, $r_{S}\left(z_{*}\right)$ depends on the matter density $\Omega_{M}$ through the expansion rate and the recombination redshift. For a baryon density $\Omega_{b} h^{2} \simeq 0.02$,

\footnotetext{
1 Institute of Cosmology and Gravitation, Mercantile House, Hampshire Terrace, University of Portsmouth, Portsmouth, UK.

2 Steward Observatory, University of Arizona, Tucson, AZ.

3 Department of Astronomy, The Ohio State University, Columbus, $\mathrm{OH}$.

4 Institute for Cosmic Ray Research, University of Tokyo, Kashiwa, Japan.

5 Department of Physics and Astronomy, The Johns Hopkins University, Baltimore, MD.

6 Institute for Astronomy, University of Hawaii, Honolulu, HI.

7 Department of Astronomy and Astrophysics, Pennsylvania State University, University Park, PA.

8 Department of Physics, Drexel University, Philadelphia, PA.

9 Department of Astronomy, Case Western Reserve University, Cleveland, OH.

${ }_{11}$ Department of Astrophysical Sciences, Princeton University, Princeton, NJ.

11 Apache Point Observatory, Sunspot, NM.

12 Department of Physics and Astronomy, University of Pittsburgh, Pittsburgh, PA.

13 Astronomy Centre, University of Sussex, Falmer, Brighton, UK.

14 SUPA; Institute for Astronomy, University of Edinburgh, Royal Observatory, Blackford Hill, Edinburgh, UK.
}

we can approximate $c_{S} \simeq 0.90 c / \sqrt{3}$. Inserting $z_{*}=1100$ and $a_{\mathrm{eq}}=\left(23900 \Omega_{M} h^{2}\right)^{-1}$ gives $r_{S}\left(z_{*}\right)=109 h^{-1} \mathrm{Mpc}$ for $\Omega_{M}=$ 0.24 and $h=0.73$.

In real space this leads to a peak in the correlation function at $r_{S}\left(z_{*}\right)$. In Fourier space this process leads to oscillations in the power spectrum in the same way that the transform of a top-hat function yields a sinc function. The wavelength of these oscillations for $\Omega_{M}=0.24$ and $h=0.73$ is $k_{S}=2 \pi / 109=0.06 h \mathrm{Mpc}^{-1}$. Numerical simulations have shown that a number of subtle corrections are required to this simple picture, although these corrections do not significantly affect the underlying important physics. On large scales, there is a phase shift in the position of the oscillations due to the contribution of the baryons to the drag epoch (Eisenstein \& Hu 1998). We must also consider the damping of the oscillations on small scales at high redshift (Silk 1968), and when modeling the oscillations at low redshifts, due to structure formation (Eisenstein et al. 2007).

The resulting acoustic peaks in the cosmic microwave background (CMB) have now been observed with extreme precision (Hinshaw et al. 2007; Spergel et al. 2007). However, interpreting the observed angular separation of these peaks in terms of the physics of the early universe requires knowledge of the angular diameter distance to the last scattering surface; in the context of flat $\Lambda$ cosmological models this leads to a parameter degeneracy between the matter density $\Omega_{M}$ and the Hubble constant $h$, and models with the same value of $\Omega_{M}^{0.275} h$ have the same projected acoustic horizon scale (Percival et al. 2002; Page et al. 2003; Spergel et al. 2007.) Throughout this paper a "flat $\Lambda$ " cosmological model implies a universe that is spatially flat with a timeindependent "dark energy" component, i.e., a cosmological constant. Theoretically, we should expect the oscillations to survive in the galaxy power spectrum (Meiksin et al. 1999; Springel et al. 2005; Seo \& Eisenstein 2005; White 2005; Eisenstein et al. 2007), and the effects of baryons have been previously detected on large scales in the clustering of galaxies (Percival et al. 2001; Miller et al. 2001; Cole et al. 2005; Eisenstein et al. 2005; Huetsi 2006). These low-redshift observations have a different dependence on the cosmological distance-redshift relation compared with the CMB because of the different angular and radial projection 
of these features. By comparing the two observations we can therefore probe the cosmological expansion history in addition to the physics of the BAO production. In this paper we use the BAO measured in the SDSS DR5 galaxy sample to set tight, clean constraints on the cosmological matter density.

\section{MEASURING THE SDSS POWER SPECTRUM}

The data and method used for calculating the redshift-space power spectrum of the latest SDSS sample are described in detail in Percival et al. (2007). In this section we summarize this information, paying particular attention to important details for the BAO detection. The SDSS (York et al. 2000; Adelman-McCarthy et al. 2006; Blanton et al. 2003; Fukugita et al. 1996; Gunn et al. 1998, 2006; Hogg et al. 2001; Ivezic et al. 2004; Pier et al. 2003; Smith et al. 2002; Stoughton et al. 2002; Tucker et al. 2006) Data Release 5 (DR5) sample represents the largest volume of the universe that has been mapped to date. In total we have 522,280 galaxy spectra, with 465,789 of those spectra being main galaxies (Strauss et al. 2002) selected to a limiting magnitude $r<17.77$, or $r<17.5$ in a small subset of the early data from the survey. The remaining 56,491 galaxies are luminous red galaxies (LRGs; Eisenstein et al. 2001), which form an extension to the survey to higher redshifts $0.3<z<0.5$; this extension covers most of the volume mapped. Of the main galaxies, 21,310 are also classified as LRGs, so our sample includes 77,801 LRGs in total. In this paper and its companion (Percival et al. 2007), we analyze the combined sample of main galaxies and LRGs, thereby including correlations between the two samples. Although the main galaxy sample contains significantly more galaxies than the LRG sample, the LRG sample covers more volume, and therefore contains almost all of the cosmological information. However, as shown in Figure 8 of Percival et al. (2007), the contribution of pairs of galaxies, where one galaxy is a main sample galaxy and one is a LRG, are not negligible compared with LRG-LRG pairs, justifying the added complexity of an analysis using both data sets.

The sample now contains $60 \%$ more LRGs than considered in the first measurement of BAO in the SDSS LRG sample (Eisenstein et al. 2005); with the increased precision due to this increase in volume and the main-LRG pairs of galaxies, we can now attempt to derive constraints on the matter density that do not rely on the overall shape of the cold dark matter (CDM) power spectrum, but only on the peak locations relative to a smooth underlying spectrum. Although ignoring the overall shape removes information, we gain in robustness due to the increased simplicity of the physics producing the cosmological constraint; measurements of the matter density from the overall shape of the galaxy power spectrum are potentially affected by galaxy bias (Percival et al. 2007), or the form of the fluctuation spectrum from the inflationary model.

The redshift-space clustering power spectrum of this sample has been calculated using a Fourier-based technique (Feldman et al. 1994; Percival et al. 2004). This method uses a simple model for the relative bias of galaxies to remove effects in the power spectrum due to pairs of galaxies with different expected clustering amplitudes. The power spectrum is recalculated for 31 flat $\Lambda$ cosmological models, with matter density $0.1 \leq \Omega_{M} \leq 0.4$ and $\Delta \Omega_{M}=0.01$. For each of these cosmological models, we have created 2000 lognormal catalogs (using the method described in Cole et al. 2005) with power spectra calculated using a linear CDM model with parameters chosen to approximately match the amplitude and shape of the recovered power for $0.01<k<$ $0.15 h \mathrm{Mpc}^{-1}$. These power spectra are used to calculate a covariance matrix for each model, although we then minimize the effect of Monte Carlo noise in each element in the set of covariance matrices as a function of $\Omega_{M}$ by smoothing using a
4 node cubic spline with nodes at $\Omega_{M}=0.1,0.2,0.3,0.4$. This ensures a smooth progression in the error estimation over the set of cosmological models. The convolving effect of the survey geometry on the power spectrum has also been quantified by spline fitting the Fourier transform of the window function. This fit is used to smooth all models before comparison with the data.

\section{MODELING THE BARYON ACOUSTIC OSCILLATIONS}

We model the true galaxy power spectrum on scales $0<k<$ $0.3 h \mathrm{Mpc}^{-1}$ with a two-component model. The overall shape was matched using a cubic spline fit with 8 nodes separated by $\Delta k=0.05 h \mathrm{Mpc}^{-1}$ and $0.025 \leq k \leq 0.375 h \mathrm{Mpc}^{-1}$, and an additional node at $k=0.001 h \mathrm{Mpc}^{-1}$. This smooth model was then modulated by a higher frequency component constructed as follows: the sinusoidal BAO term in a standard CDM transfer function was estimated for the parameters to be tested from numerical fits (Eisenstein \& Hu 1998; Eisenstein et al. 2007), including a damping term to approximately correct for nonlinear structure formation, and the multiplicative effect on a CDM power spectrum was isolated. This multiplicative term was then applied to the smooth cubic spline power spectrum rather than a CDM model. The model power spectra are adjusted for the effects of the survey geometry by convolving with the appropriate window function and correcting for our lack of knowledge about the true mean density of galaxies by subtracting a multiple of the Fourier window function from the model power so that $P(0)=0$ (see Percival et al. 2007 for details).

This procedure separates the physics of the BAO from that governing the overall shape of the power spectrum, including both cosmological and galaxy formation effects, and ensures that the cosmological constraints presented in this paper only come from the BAO and not from the additional physics encoded in the power spectrum. In particular, forming a model power spectrum in this way allows for nonlinear effects and galaxy bias to change the overall shape of the power spectrum, and damp the oscillations on small scales. The model does not allow the BAO scale to change, although it is worth emphasizing that the model can lead to an apparent change in the positions of the peaks and troughs in the model power spectra caused by the addition of a tilted smooth power spectrum component (for example the 1 halo term in the halo model), or multiplication by such a smooth component. Recent theoretical models of BAO in galaxy power spectra find such an apparent change in the positions of the peaks and troughs (Seo \& Eisenstein 2005; Smith et al. 2007), but have not shown evidence for a change in the BAO scale. Because we split into the $\mathrm{BAO}$ and a smooth component, any such apparent shift in the observed BAO scale is removed by our analysis method. Extra multiplicative or additive low-frequency power spectrum components will change the BAO damping, which will be a function of the luminosity of the galaxy sample. For a combined sample of galaxies with different luminosity such as analyzed in this paper, the form and amplitude of the small-scale BAO damping will depend on the details of the galaxy sample. However, the damping does not have a significant effect on our results, so our results are expected to be robust to such complexities.

Because of the large volume observed, the Fourier modes are not strongly correlated; correlations between modes drop to $<0.33$ for $\Delta k>0.01 h \mathrm{Mpc}^{-1}$. The correlation scale is therefore significantly smaller than the BAO wavelength. However, we calculate the likelihood of the data given each model to be tested assuming that the data are drawn from a multivariate Gaussian distribution with covariance matrix calculated as described in $\S 2$ (including the effect of the change in the determinant as a function 


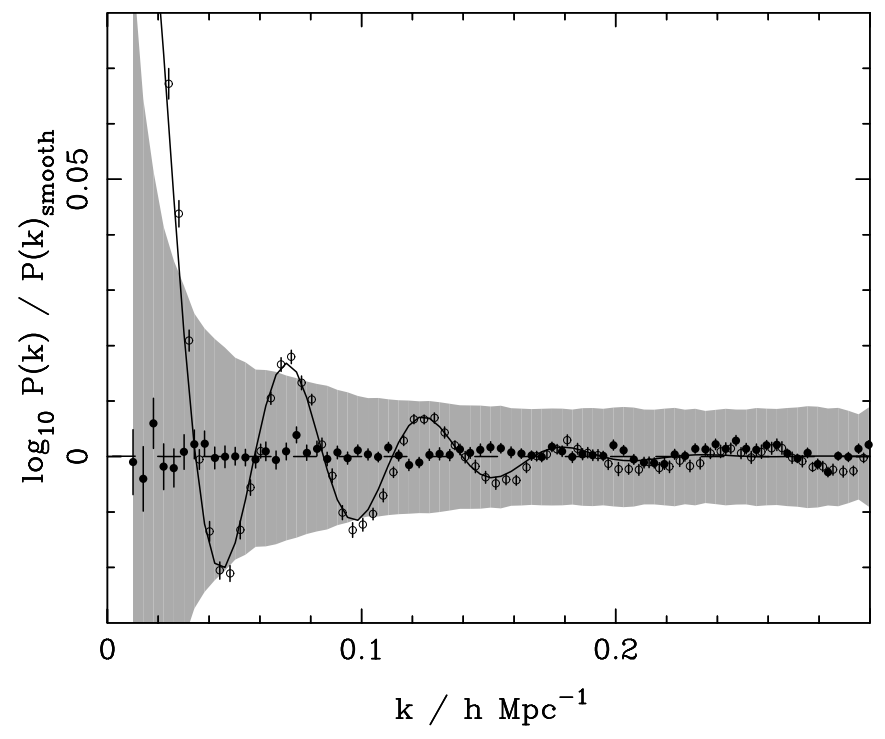

FIG. 1.-Results from fitting to 200 mock linear CDM power spectra calculated assuming $h=0.73, \Omega_{M}=0.24$, and $n_{s}=0.96$ with our two component spline+BAO model. We plot the average ratio between input power and spline fit from 100 mock CDM power spectra with no baryon oscillations (open circles with $1 \sigma$ errors), and 100 mocks with baryon oscillations assuming $\Omega_{b} / \Omega_{M}=$ 0.174 ( filled circles with $1 \sigma$ errors). The expected residual in each case is shown by the solid lines. From these fits we find that the difference between input and recovered power $\Delta P(k) / P(k)<0.015$ for $0.01<k<0.3 h \mathrm{Mpc}^{-1}$, a level well below the current experimental error (gray shaded region).

of $\Omega_{M}$ ). For each cosmological model tested, we calculate the maximum likelihood obtained after varying the values of the smooth spline function at the nine nodes for models, where we include or exclude the BAO model. Ideally, excluding the BAO, we would find that the likelihood does not change with the cosmological model. However, because the data change with $\Omega_{M}$, we find small variations in the likelihood even when only fitting a smooth curve to the power spectra. To remove this "noise" we only consider the ratio of the different likelihoods including and excluding the BAO signal.

This method relies on the spline curve being able to fit the power spectrum shape. In order to test this, we have fitted two sets of 100 linear CDM power spectra (calculated using the fits of Eisenstein \& Hu 1998 assuming $h=0.73, \Omega_{M}=0.24$, and $n_{s}=$ $0.96)$ with our combined spline+BAO model. The first set of mock data contained BAO with $\Omega_{b} / \Omega_{M}=0.174$, while the second set had no BAO $\left(\Omega_{b}=0\right)$. Noise was added to the mock power spectra drawn from a multivariate Gaussian distribution with covariance matrix matched to the real data, and the power spectra were convolved with the window function of the survey (for a cosmological distance model with $\Omega_{M}=0.24$ ). For the combined spline $+\mathrm{BAO}$ model that we fit to these data, we have assumed that there is no small-scale damping, and have fixed the cosmological parameters at the input values. The spline curves are then allowed to vary to fit the data, and the average residuals between the mock power and the spline fit are plotted in Figure 1, compared to the expected residuals. The results match those expected at a level well below the error on the power spectrum; this and the acceptable average $\chi^{2}$ values of the fits (64.2 and 66.2 for the sets of models with and without BAO, given 64 dof) show that the spline+BAO model can match the features expected in a linear CDM power spectrum.

Finally, we end this section with a brief discussion on the node separation chosen for the spline fit. If too many nodes are chosen, then the spline fit can itself match the BAO, leading to a small

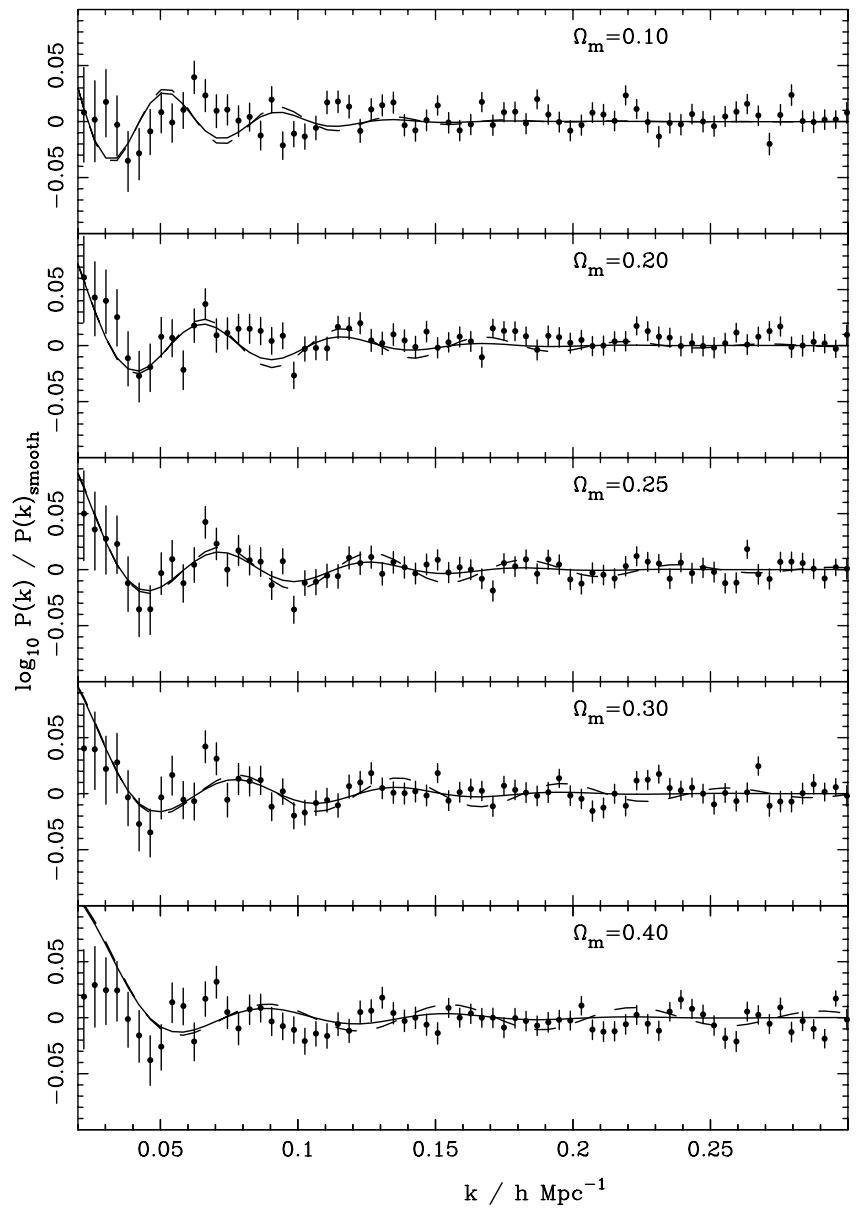

FIG. 2.- Ratio of the power spectra calculated from the SDSS to the smooth cubic spline fit that we use to model the overall shape of the measured power spectra ( filled circles with $1 \sigma$ errors). Data are plotted using five flat $\Lambda$ cosmological models to convert from redshift to comoving distance, with matter densities given in each panel. For comparison, in each panel we also plot the BAO predicted by a CDM model with the same matter density, $h=0.73$, and a $17 \%$ baryon fraction (solid lines). The dashed lines show the same models without the low-redshift small-scale damping term. As can be seen, the observed oscillations approximately match those predicted by this model for $0.2 \leq \Omega_{M} \leq 0.3$, but fail for higher or lower matter densities.

likelihood ratio between models with and without $\mathrm{BAO}$ even if $\mathrm{BAO}$ are strong in the data. Conversely, if too few nodes are chosen, the addition of BAO to the model will not necessarily match high-frequency features in the observed power spectrum and might instead simply help to fit the overall shape. The node spacing adopted in this work was carefully chosen based on the analysis of mock power spectra and from the results of the fits to the SDSS power spectra; with the chosen separation, no evidence was observed for low-frequency residuals from any of the SDSS power spectra fits, with or without BAO.

\section{RESULTS}

In Figure 2 we plot the measured power spectra determined assuming five different values of the matter density divided by the best-fit smooth cubic spline fit (solid circles). The spline fits were calculated after fitting the data, including a possible BAO contribution with fixed $\Omega_{b} / \Omega_{M} \sim 0.17$ and $h=0.73$, and the appropriate $\Omega_{M}$. These data are compared with the BAO model, and show that the model and data match only if $0.2 \lesssim \Omega_{M} \lesssim 0.3$, assuming $\Omega_{b} / \Omega_{M} \sim 0.17$ and $h=0.73$. For $\Omega_{M}=0.26$, the baryon oscillations are required with a likelihood ratio $2 \ln \mathcal{L}=9.02$, 


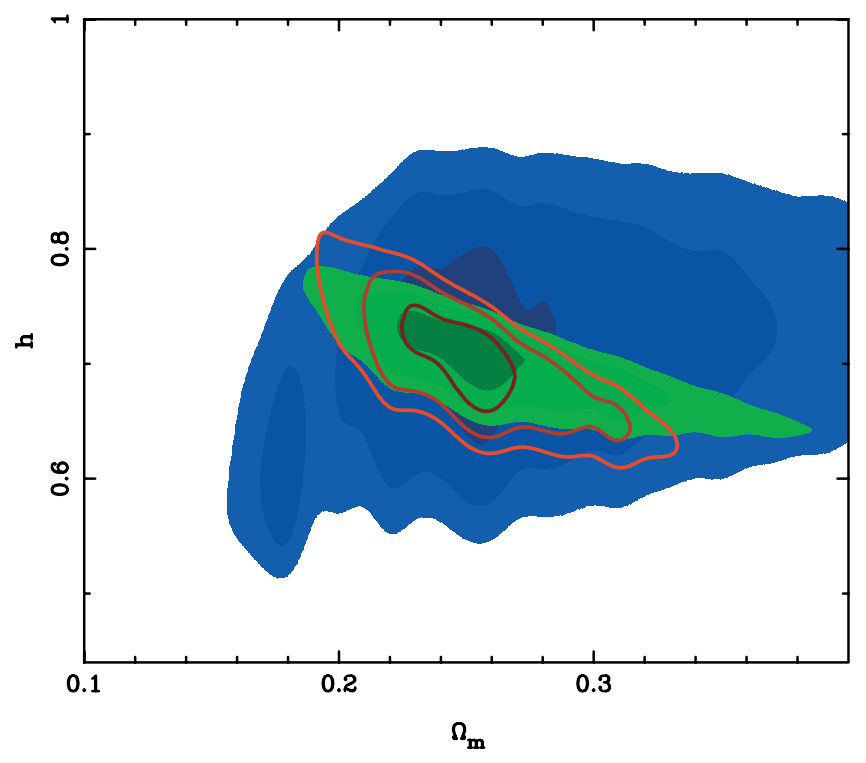

FIG. 3.-Likelihood contours in the $h-\Omega_{M}$ plane, derived from measurements of the BAO observed in the SDSS combined with constraints from other cosmological data. The intensities are separated by $-2 \ln \mathcal{L}=2.3,6.0,9.2$, corresponding to two-parameter confidence of $68 \%, 95 \%$, and $99 \%$ for a Gaussian distribution. The blue shaded region shows the constraints for flat $\Lambda$ cosmologies combining the SDSS BAO data with a low-redshift constraint on $h=0.72 \pm 0.08$ for flat $\Lambda$ cosmologies. The green region shows the combination of the SDSS constraint with the 3 yr $W M A P$ constraints on $\Omega_{M}^{0.275} h$ and $\Omega_{b} h^{2}$, again for flat $\Lambda$ cosmologies. The overlaid red contours were calculated by instead combining with a constraint on $\Omega_{M} h^{2}$ from the peak heights in the CMB together with the constraint on $\Omega_{b} h^{2}$. This relaxes the assumption of a flat $\Lambda$ cosmology, by removing the dependence on the distance to the last scattering surface. There is still a dependence on the comoving distance-redshift relation over the survey, but observations of Type Ia supernovae constrain this to be close to that expected for a flat $\Lambda$ cosmology. The degeneracies in the $h-\Omega_{M}$ plane induced by different cosmological observations are discussed at length in Tegmark et al. (2006).

corresponding to a $99.74 \%$ confidence of detection (3.0 $\sigma$ assuming Gaussianity).

Because the amplitude of the BAO depends on the fraction of matter that is baryonic, the SDSS observations constrain the baryon fraction, although this constraint is weak compared to constraints on $\Omega_{b} h^{2}$ from CMB observations. The scale of the BAO depends on $\Omega_{M}$ and $h$, so we need one other piece of information about a combination of these parameters to break this degeneracy and measure $\Omega_{M}$. We consider three options, yielding the three sets of likelihood contours in the $h-\Omega_{M}$ plane that are shown in Figure 3. First, we combine our low-redshift BAO measurement with the HST Key Project (Freedman et al. 2001) estimate of the Hubble parameter $h=0.72 \pm 0.08$ ( $1 \sigma$ errors). This gives $\Omega_{M}=$ $0.256_{-0.029}^{+0.049}$ (all the error bars quoted in this paper span the $68 \%$ confidence interval). Here we have marginalized over the uncertainty in $h$ (assumed to be Gaussian) and over a uniform prior on the baryon density $0.008<\Omega_{b} h^{2}<0.034$. This estimate of $\Omega_{M}$ assumes a flat $\Lambda$ cosmological model, but dark energy and spatial curvature affect the result only through their influence on the distance-redshift relation for the galaxies within our sample. Measurements of Type Ia supernovae already demonstrate that this relation is close to that expected for a flat $\Lambda$ universe; we estimate that the allowable residual effect on $\Omega_{M}$ is at most \pm 0.02 .

As an alternative to direct $h$ measurement, we consider the combination of our low-z, redshift-space measurement of the BAO scale with the angular scale of the acoustic oscillations measured in the CMB. The 3 yr WMAP data (Spergel et al. 2007) yields the con- straint $\Omega_{M}^{0.275} h=0.492_{-0.017}^{+0.008}$ after marginalizing over the scalar spectral index and the baryon density. Combined with our BAO measurement, this yields $\Omega_{M}=0.256_{-0.024}^{+0.029}$ and $h=0.709_{-0.027}^{+0.022}$. For simplicity, we have made the good but not perfect approximation that the WMAP baryon density constraint, $100 \Omega_{b} h^{2}=$ $2.233_{-0.091}^{+0.072}$, is independent of the acoustic scale constraint. Relaxing the baryon density constraint by a factor of 4 makes essentially no difference to either the best-fit $\Omega_{M}$ or the error bars. This second determination of $\Omega_{M}$ relies more heavily on the assumption of a flat $\Lambda$ universe, since space curvature or a different dark energy component would change the distance to the last scattering surface and hence the angular scale of the CMB acoustic peaks.

We can weaken the dependence on the assumption of a flat $\Lambda$ cosmology by instead combining our low-redshift BAO measurement with the WMAP constraint on the matter density, $\Omega_{M} h^{2}=$ $0.1268_{-0.0095}^{+0.0072}$. This constraint comes from the relative heights of the CMB acoustic peaks, so the physics that underlies it is somewhat different and somewhat more degenerate with the parameters of the inflationary fluctuation spectrum. The dependence on the assumption of a flat $\Lambda$ cosmology is weaker because the relative CMB peak heights only depend on the distance to the last scattering surface through the weak, and predominantly large angle, integrated Sachs-Wolfe effect. In addition, we have already argued that observations of Type Ia supernovae constrain the distanceredshift relation for the low-redshift galaxies to be close to a flat $\Lambda$ cosmology (leaving a residual effect $\Delta \Omega_{M}$ at most \pm 0.02 ). The direction of the $\Omega_{M} h^{2}$ constraint in the $h-\Omega_{M}$ plane is more complementary to the low-redshift BAO observations for measuring $\Omega_{M}$ compared with the CMB peak positions, and this combination yields $\Omega_{M}=0.256_{-0.022}^{+0.020}$, again marginalizing over the $W M A P$ baryon density constraint.

\section{DISCUSSION}

In a separate paper, Tegmark et al. (2006) carry out full multiparameter fits to the $\mathrm{LRG}$ power spectrum and WMAP data over a broader CDM model space, drawing on both the BAO measurement and the broadband shape of the power spectrum. The more focused analysis presented in this paper is complementary, obtaining constraints with minimal dependence on detailed cosmological assumptions. Within the context of flat $\Lambda$ models, the measurement of the matter density, $\Omega_{M}=0.256_{-0.024}^{+0.029}$, from the locations of the acoustic oscillations (the second combination above) is especially "clean," relying on a single piece of wellunderstood physics. In particular, we have decoupled constraints from the BAO with constraints from the overall shape of the power spectrum on the same scales. As demonstrated in Percival et al. (2007), the overall shape of the power spectrum, even on scales where the matter clustering has not deviated strongly from a linear CDM model, is dependent on the galaxy population studied. It is therefore imperative to accurately model galaxy bias before robust cosmological constraints can be derived from such observations. By simply considering the BAO in this paper we avoid this complexity.

Given the different physics probed, the consistency of the three $\Omega_{M}$ estimates calculated with different additional information itself provides support for the assumptions of a flat universe with a cosmological constant. With larger samples and a wider redshift range, the BAO "standard ruler" can be used to test these assumptions at high precision through the comoving distanceredshift relation (Blake \& Glazebrook 2003; Seo \& Eisenstein 2003). Forthcoming surveys have been designed to exploit this effect. It is clear that we are entering an era where BAO offer an extremely attractive route to strong, robust cosmological constraints. 
W. J. P. is grateful for support from a PPARC fellowship, and R. C. N. for a EU Marie Curie Excellence Chair.

Funding for the SDSS and SDSS-II has been provided by the Alfred P. Sloan Foundation, the Participating Institutions, the National Science Foundation, the US Department of Energy, the National Aeronautics and Space Administration, the Japanese Monbukagakusho, the Max Planck Society, and the Higher Education Funding Council for England. The SDSS web site is http://www.sdss.org/.

The SDSS is managed by the Astrophysical Research Consortium for the Participating Institutions. The Participating Institutions are the American Museum of Natural History, Astrophysical
Institute Potsdam, University of Basel, Cambridge University, Case Western Reserve University, University of Chicago, Drexel University, Fermilab, the Institute for Advanced Study, the Japan Participation Group, The Johns Hopkins University, the Joint Institute for Nuclear Astrophysics, the Kavli Institute for Particle Astrophysics and Cosmology, the Korean Scientist Group, the Chinese Academy of Sciences (LAMOST), Los Alamos National Laboratory, the Max-Planck-Institute for Astronomy (MPIA), the Max-Planck-Institute for Astrophysics (MPA), New Mexico State University, The Ohio State University, University of Pittsburgh, University of Portsmouth, Princeton University, the United States Naval Observatory, and the University of Washington.
Adelman-McCarthy, J., et al. 2006, ApJS, 162, 38

Bashinsky, S., \& Bertschinger, E. 2001, Phys. Rev. Lett., 87, 081301 2002, Phys. Rev. D, 65, 123008

Blake, C., \& Glazebrook, K. 2003, ApJ, 594, 665

Blanton, M. R., Lin, H., Lupton, R. H., Maley, F. M., Young, N., Zehavi, I., \& Loveday, J. 2003, AJ, 125, 2276

Bond, J. R., \& Efstathiou, G. 1984, ApJ, 285, L45 . 1987, MNRAS, 226, 655

Cole, S., et al. 2005, MNRAS, 362, 505

Eisenstein, D. J., \& Hu, W. 1998, ApJ, 496, 605

Eisenstein, D. J., Seo, H.-J., \& White, M. 2007, ApJ, submitted (astro-ph/ 0604361)

Eisenstein, D. J., et al. 2001, AJ, 122, 2267 2005, ApJ, 633, 560

Feldman, H. A., Kaiser, N., \& Peacock, J. A. 1994, ApJ, 426, 23

Freedman, W. L., et al. 2001, ApJ, 553, 47

Fukugita, M., Ichikawa, T., Gunn, J. E., Doi, M., Shimasaku, K., \& Schneider, D. P. 1996, AJ, 111, 1748

Gunn, J. E., et al. 1998, AJ, 116, 3040 2006, AJ, 131, 2332

Hinshaw, G., et al. 2007, ApJ, submitted (astro-ph/0603451)

Hogg, D. W., Finkbeiner, D. P., Schlegel, D. J., \& Gunn, J. E. 2001, AJ, 122, 2129

Holtzman, J. A. 1989, ApJS, 71, 1

Hu, W., \& Sugiyama, N. 1995, ApJ, 444, 489

Huetsi, G. 2006, A\&A, 449, 891

\section{REFERENCES}

Ivezic, Z., et al. 2004, Astron. Nachr., 325, 583

Meiksin, A., White, M., \& Peacock, J. A. 1999, MNRAS, 304, 851

Miller, C. J., Nichol, R. C., \& Batuski, D. J, 2001, ApJ, 555, 68

Page, L., et al. 2003, ApJS, 148, 233

Peebles, P. J. E., \& Yu, J. T. 1970, ApJ, 162, 815

Percival, W. J., Verde, L., \& Peacock, J. A. 2004, MNRAS, 347, 645

Percival, W. J., et al. 2001, MNRAS, 327, 1297

- 2002, MNRAS, 337, 1068

Percival, W. J., et al. 2007, ApJ, in press (astro-ph/0608636)

Pier, J. R., et al. 2003, AJ, 125, 1559

Seo, H.-J., \& Eisenstein, D. J. 2003, ApJ, 598, 720 2005, ApJ, 633, 575

Silk, J. 1968, ApJ, 151, 459

Smith, J. A., et al. 2002, AJ, 123, 2121

Smith, R. E., Scoccimarro, R., \& Sheth, R. K. 2007, Phys. Rev. D, submitted (astro-ph/0609547)

Spergel, D. N, et al. 2007, ApJ, submitted (astro-ph/0603449)

Springel, V., et al. 2005, Nature, 435, 629

Stoughton, C., et al. 2002, AJ, 123, 485

Strauss, M. A., et al. 2002, AJ, 124, 1810

Sunyaev, R. A., \& Zel'dovich, Ya. B. 1970, Ap\&SS, 7, 3

Tegmark, M., et al. 2006, Phys. Rev. D, 74, 123507

Tucker, D., et al. 2006, Astron. Nachr., 327, 821

White, M. 2005, Astropart. Phys., 24, 334

York, D. G., et al. 2000, AJ, 120, 1579 